\title{
Double Dissociation of the Dorsomedial and Dorsolateral Striatal Control Over the Acquisition and Performance of Cocaine Seeking
}

\author{
Jennifer E Murray*,1,2, David Belin ${ }^{2,3}$ and Barry J Everitt ${ }^{1,2}$ \\ 'Department of Experimental Psychology, University of Cambridge, Cambridge, UK; ${ }^{2}$ INSERM European Associated Laboratory, Psychobiology of \\ Compulsive Habits, Poitiers, France and Cambridge, UK; ${ }^{3}$ INSERM AVENIR Team, Psychobiology of Compulsive Disorders, INSERM UI084 \\ Laboratoire de Neurosciences Expérimentales et Cliniques and Université de Poitiers, Poitiers, France
}

\begin{abstract}
The present study investigated the involvement of dopamine-dependent mechanisms in the anterior dorsolateral (aDLS) and posterior dorsomedial (pDMS) striatum during the early- and late-stage performance of cocaine-seeking behavior. Rats were trained to selfadminister cocaine under continuous reinforcement (fixed-ratio I, FRI) with a 20-s light conditioned stimulus (CS) presented contingently upon each infusion. After a week, rats were challenged by a change in contingency to seek cocaine during a 15-min period uninfluenced by cocaine during which each response was reinforced by a I-s CS presentation. Dopamine transmission blockade by intracranial infusions of $\alpha$-flupenthixol only in the pDMS, but not in the aDLS, dose dependently reduced performance of cue-controlled cocaine seeking at the early stage of self-administration. One cohort of rats was then trained with increasing response requirements until completing I 5 sessions under a second-order schedule [FII 5(FR I0:S)] so that cocaine-seeking performance became well established. At this stage, intra-aDLS, but not pDMS, $\alpha$-flupenthixol infusions dose dependently reduced active lever presses. The second cohort of rats continued to self-administer cocaine under the FRI schedule such that their drug intake was matched to the late-stage performance group. $\alpha$-Flupenthixol in the pDMS, but not in the aDLS, again prevented the performance of cocaine seeking. These results show that dopamine transmission in the PDMS is required for initial performance of goal-directed cocaine seeking, and that its role is ultimately subverted and devolves instead to the aDLS only following training with high rates of cocaine-seeking behavior, supporting the theory of dynamic shifts in the striatal control over cocaine seeking between goal-directed and habitual performance.

Neuropsychopharmacology (2012) 37, 2456-2466; doi:I0.1038/npp.2012.104; published online 27 June 2012
\end{abstract}

Keywords: cocaine self-administration; conditioned reinforcement; dopamine; dorsolateral striatum; dorsomedial striatum; habit formation

\section{INTRODUCTION}

It is well established that the ventral striatum, including the nucleus accumbens (Acb), has a key role in drug selfadministration by mediating pavlovian influences on instrumental seeking behavior (Cardinal et al, 2002; Wyvell and Berridge, 2000) as well as mediating the reinforcing effects of stimulant drugs through its dopaminergic innervation (Wise, 2004). However, it has proven more problematic to define the neural basis of the acquisition of instrumental responding for addictive drugs because it is difficult to disentangle the neural control of instrumental conditioning from their rewarding and motor effects.

*Correspondence: Dr JE Murray, Department of Experimental Psychology, University of Cambridge, Cambridge CB2 3EB, UK, Tel: + 44 (0) 1223 765285, Fax: + 44 (0) 1223733564 , E-mail: jem98@cam.ac.uk

Received 26 March 2012; revised 21 May 20 12; accepted 31 May 2012
Whereas the acquisition of cocaine self-administration is impaired by manipulations that diminish the reinforcing effects of the drug, such as Acb dopamine depletion (Roberts and Koob, 1982) and dopamine receptor blockade (Robledo et al, 1992), it is not prevented by specific lesions of either the Acb core (AcbC) or shell (AcbS) (Ito et al, 2004).

By contrast, AcbC lesions impair the acquisition of cuecontrolled cocaine seeking, as measured under a secondorder schedule of reinforcement, by disrupting the control over instrumental responses by stimuli associated with the drug acting as conditioned reinforcers (Ito et al, 2004), and weakening the ability to tolerate delays to primary reinforcement (Cardinal et al, 2001). However, when cocaine-seeking behavior is overtrained, its locus of control devolves to the anterior dorsolateral striatum (aDLS). Thus, well-established cocaine seeking is associated with increased extracellular dopamine in the aDLS, but not in the AcbC or AcbS (Ito et al, 2000), while being also greatly decreased by dopamine receptor blockade in the aDLS, but 
not in the AcbC (Vanderschuren et al, 2005). Moreover, in a cocaine seeking-taking chained schedule in which pressing a 'seeking' lever allows access to a 'taking' lever (Olmstead et al, 2001), inactivation of the aDLS restored sensitivity to reinforcer devaluation after overtraining, thereby reinstating cocaine seeking as goal-directed in rats that had developed a cocaine-seeking habit (Zapata et al, 2010).

Although consistent with evidence implicating the aDLS in habit learning (Thorn et al, 2010; Yin et al, 2004) and the hypothesis that a shift from goal-directed action-outcome (A-O) to habitual stimulus-response (S-R) control over drug seeking depends upon progressive engagement of aDLS dopamine transmission (Everitt et al, 2008), these data do not identify the striatal mechanisms underlying the acquisition of goal-directed drug seeking. The posterior dorsomedial striatum (pDMS), a region not previously investigated in the context of drug self-administration, has, however, been shown to be important for acquisition of responding for food (Yin et al, 2004). Lesions of the pDMS result in insensitivity to reinforcer devaluation and contingency degradation (Yin et al, 2005), while dopamine and extracellular signal-regulated kinase (ERK) signalling in the pDMS are required for acquisition of A-O instrumental action (Shiflett $e t$ al, 2010). This body of evidence suggests that the shift in the striatal locus of control in the transition from goal-directed to habitual cocaine seeking may not only be dependent on the circuitry linking the ventral to the dorsolateral striatum via serial connections with midbrain dopamine neurons (Haber, 2003; Ikemoto, 2007; Nauta et al, 1978). It may also depend upon a progressive, dopaminedependent shift within the dorsal striatal territories from the medial-posterior to the lateral-anterior area. The former, as with the AcbC, receives afferents from the ventral tegmental area (VTA) dopamine neurons, whereas the latter receives inputs from the anterior substantia nigra (Gerfen et al, 1987). We therefore tested the hypothesis that pDMS dopamine-dependent mechanisms are involved in the early performance of cocaine seeking, but subsequently become overshadowed by dopaminergic mechanisms in the aDLS when the behavior is well established. We manipulated dopamine transmission in the pDMS and aDLS at different stages of cocaine seeking in a procedure that captures key features of real-world foraging for drugs, including delays to reinforcement and a pivotal role of drug-associated stimuli (Everitt and Robbins, 2000).

\section{MATERIALS AND METHODS}

\section{Animals}

Male Lister Hooded rats (Charles River Laboratories, Kent, UK) weighing $353 \pm 6 \mathrm{~g}$ at the time of surgery were housed as described previously (Murray et al, 2012; see Supplementary General Materials and Methods). Experiments were conducted in accordance with the United Kingdom 1986 Animals (Scientific Procedures) Act, Project License 80/ 2234.

\section{Drugs}

Cocaine hydrochloride (Macfarlan-Smith, Edinburgh, UK) was dissolved in sterile $0.9 \%$ saline. $\alpha$-Flupenthixol (Sigma-
Aldrich, Poole, UK) was dissolved in double-distilled water. Drug doses are reported in the salt form.

\section{Surgery}

Rats were anesthetized and implanted with a pre-fabricated intravenous jugular catheter as described previously (Murray et al, 2012; see Supplementary General Materials and Methods). They were then positioned in a stereotaxic frame (David Kopf Instruments, Tujunga, CA, USA) and were implanted bilaterally with 22-gauge guide cannulae (Plastics One, Roanoke, VA, USA) positioned to lie $2 \mathrm{~mm}$ above either the aDLS (anteroposterior (AP) +1.2, mediolateral $(\mathrm{ML}) \pm 3$, dorsoventral (DV) -3 ; Belin and Everitt, 2008) or the pDMS (AP-0.4, ML $\pm 2.6, \mathrm{DV}-2.5$; Lex and Hauber, 2010) infusion targets. AP and ML coordinates were measured from bregma, DV coordinates from the skull surface, with the incisor bar set at $-3.3 \mathrm{~mm}$ (Paxinos and Watson, 1998). Cannulae were held in place using dental acrylic anchored to stainless steel screws tapped into the frontal and parietal bones of the skull. Obturators (Plastics One) were placed in the cannulae to maintain patency. Rats were treated daily from the day before to 7 days after surgery with $10 \mathrm{mg} / \mathrm{kg}$ subcutaneous of the antibiotic, Baytril (Bayer). Catheters were flushed daily with $0.2-$ $0.4 \mathrm{ml}$ of sterile saline mixed with heparin $(20 \mathrm{U} / \mathrm{ml}$; Wockhardt UK, Wrexham, UK) to maintain patency.

\section{Apparatus}

Experiments were conducted using twelve standard operant conditioning chambers equipped with two levers as described previously (Murray et al, 2012). For details, see Supplementary General Materials and Methods.

\section{Procedures}

A schematic representation of the full training procedure for experiments 1 and 2 is provided in the top portion of Figure 1a. Cocaine self-administration training sessions began 7 days following surgery. Cocaine $(0.25 \mathrm{mg} /$ infusion; $0.1 \mathrm{ml} / 5 \mathrm{~s}$ ) was available under a continuous reinforcement (ie, fixed-ratio 1, FR1) schedule in which one active lever press resulted in an infusion and initiated a 20-s time out. Each cocaine infusion was accompanied by the 20-s illumination of the cue-light (conditioned stimulus; CS) above the active lever, the houselight was extinguished and both levers were retracted. Pressing on the inactive lever had no programmed consequence but was recorded to provide an index of general activity. The maximum number of available cocaine infusions during this stage was 30 . Active and inactive lever assignment was counterbalanced.

Experiment 1: Early performance of cocaine seeking. The effects of striatal dopamine receptor blockade on early-stage cocaine seeking were tested following six training sessions under the FR1 schedule of reinforcement. Bilateral infusions of $\alpha$-flupenthixol $(0,5,10$, and $15 \mu \mathrm{g} /$ infusion in a counterbalanced, latin-square order of treatment) were made into the pDMS or aDLS loci $5 \mathrm{~min}$ before testing. During each 15min test session, every active lever press resulted in a 1-s light CS presentation, and cocaine was only delivered on the 

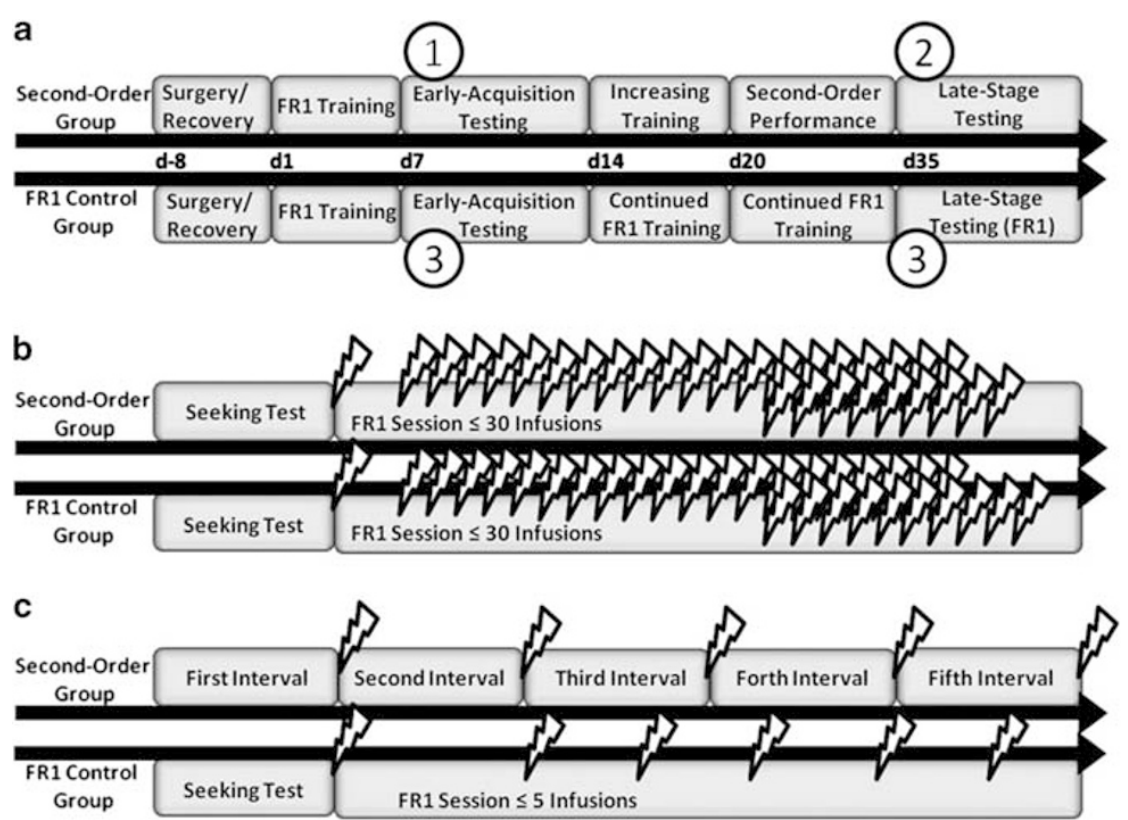

Figure I The time line of experimentation (a) for both the second-order group and the FRI control group is shown and the relationships to Experiments I, 2, and 3 are denoted. Subjects underwent intravenous and cannulae surgery a week before beginning behavioral training. For both groups, there were six sessions of FRI training followed by early-acquisition testing. From days 14 to 19, the response requirement for the second-order group was increased across sessions while the FRI control group remained on FRI training. From days 20 to 34, rats in the second-order group were maintained on the secondorder schedule, and rats in the FRI control group continued with FRI training. Late-stage testing began on day 35. Panels (b) and (c) depict early-acquisition testing and late-stage testing, respectively, for the second-order and FRI groups. In the early-acquisition tests, effects of $\alpha$-flupenthixol infusions are taken from the I5-min seeking tests. In the late-stage tests, effects of $\alpha$-flupenthixol infusions are taken from the first I5-min interval in the second-order group and from the I5-min seeking test in the FRI control group. The 'flash' indicates a cocaine infusion.

first lever press following completion of the fixed interval (FI) 15-min (formally therefore, this was the first introduction of a second-order schedule of reinforcement: [FI15(FR1:S)]). Thus, the effects of $\alpha$-flupenthixol on seeking were measured before the influence of the cocaine on a given test session. Each test session was immediately followed by a session of cocaine self-administration under FR1 (30 reinforcers over $2 \mathrm{~h}$ ) as in initial training (Figure $1 \mathrm{~b}$, top portion), and there was an additional FR1 training session between test days so as to maintain a stable cocaine-taking baseline.

Experiment 2: Maintenance of well-established cocaine seeking. On completion of tests evaluating the early performance of cocaine seeking, the response requirement was increased through the daily training sessions as follows: FR1; FR3; FR5(FR2:S); FR10(FR2:S); FR10(FR4:S); FR10 (FR6:S); FR10(FR10:S) and finally to an overall FI(FR) schedule of FI15(FR10:S) as in previous studies (eg, Belin and Everitt, 2008). Under each intermediate second-order schedule stage, completion of the unit schedule (given within parentheses) resulted in 1-s CS light presentation; cocaine infusions and the 20-s time out were given only upon completion of the overall schedule. During the final FI15(FR10:S) schedule, responding was maintained by contingent presentation of the cocaine-associated CS after 10 responses (FR10:S); cocaine was delivered on completion of the first 10 lever presses following the end of the FI $15 \mathrm{~min}$. The number of cocaine infusions per session at this stage of training was limited to 5 .
Following 15 daily sessions of responding for cocaine under FI15(FR10:S), which has been shown to result in the recruitment of aDLS dopamine-dependent control over cocaine seeking (Belin and Everitt, 2008; Ito et al, 2002; Vanderschuren et al, 2005), rats were again infused bilaterally with $\alpha$-flupenthixol $(0,5,10$, and $15 \mu \mathrm{g}$ /infusion in a counterbalanced, latin-square order of treatment) into either the aDLS or pDMS 5 min before testing (Figure 1c, top portion). Therefore, this test measured the effect of dopamine receptor blockade in aDLS and pDMS on the latestage performance, or maintenance, of the well-established cocaine-seeking response and also at a point when behavior in that session was therefore unaffected by self-administered cocaine. However, seeking responses during the second interval provided a measure of the effect of $\alpha$ flupenthixol on responding under the direct influence of cocaine infused at the end of the first seeking interval. Rats were given a training session under FI15(FR10:S) conditions between each $\alpha$-flupenthixol infusion test to ensure stable baseline levels of responding.

Experiment 3: Performance of cocaine seeking after a limited and extended cocaine-taking history. To control for the amount of cocaine self-administered and the duration of training as factors determining any shift in the striatal locus of control over responding in Experiments 1 and 2, we conducted a control experiment in which rats continued to self-administer cocaine under an FR1 schedule of reinforcement throughout training rather than having their response requirements increased to the level of the 
FI15(FR10:S) second-order schedule of reinforcement, thus equating their cocaine-taking history (Figure 1a, bottom portion). Therefore, the initial early-stage cocaine-seeking test following bilateral $\alpha$-flupenthixol infusions in the aDLS and pDMS was in fact a repetition of the early-performance test in Experiment 1 (Figure 1b, bottom portion). Following those tests, rats in this FR1 Control Group continued to selfadminister cocaine under the FR1 schedule of reinforcement such that their cocaine intake was matched to that in the extended training second-order schedule group in Experiment 2 until they had completed at least 15 sessions with a limit of 5 cocaine infusions per session. Tests of cocaine seeking in the presence of the conditioned reinforcer were then conducted as in the early-performance stage (ie, FI15(FR1:S)), each followed by a standard cocaine self-administration session under FR1 (Figure 1c, bottom portion). Rats were again given a training session between each $\alpha$-flupenthixol infusion test to ensure stable baseline levels of cocaine taking.

\section{Intrastriatal Infusions}

Intrastriatal infusions $(0.5 \mu \mathrm{l} /$ side $)$ were made via 28 -gauge steel hypodermic injectors (Plastics One) lowered to the injection sites $2 \mathrm{~mm}$ ventral to the end of the guide cannulae (ie, aDLS, $-5 \mathrm{~mm}$; pDMS, $-4.5 \mathrm{~mm}$ ). Infusions were made over $90 \mathrm{~s}$ using a syringe pump (Harvard Apparatus, Holliston, MA, USA) and were followed by a 60 -s period to allow diffusion of the infused drug or vehicle before injectors were removed and obturators were replaced. Test sessions began $5 \mathrm{~min}$ later.

\section{Histology}

At the end of the experiment, brains were processed as described in Supplementary General Materials and Methods and cannula placements mapped onto standardized coronal sections of the rat brain (Paxinos and Watson, 1998).

\section{Statistical Analyses}

Lever presses during tests were analyzed using two-way analyses of variance (ANOVAs) with Lever (active or inactive) and Dose $(0,5,10$, or $15 \mu \mathrm{g})$ as within-subject factors. Significance was set at $\alpha=0.05$. Significant interactions were analyzed further using Tukey's honestly significant difference (HSD) tests.

\section{RESULTS}

\section{Histological Assessment of Cannula Placements}

Schematic representations (Paxinos and Watson, 1998) of the infusion locations in the aDLS are shown in Figure 2a (second-order group, left panel; FR1 control group, right panel), and infusion locations in the pDMS are shown in Figure 2b (second-order group, left panel; FR1 control group, right panel). All animals included in the behavioral statistical analyses had cannulae located bilaterally within the targeted striatal areas.
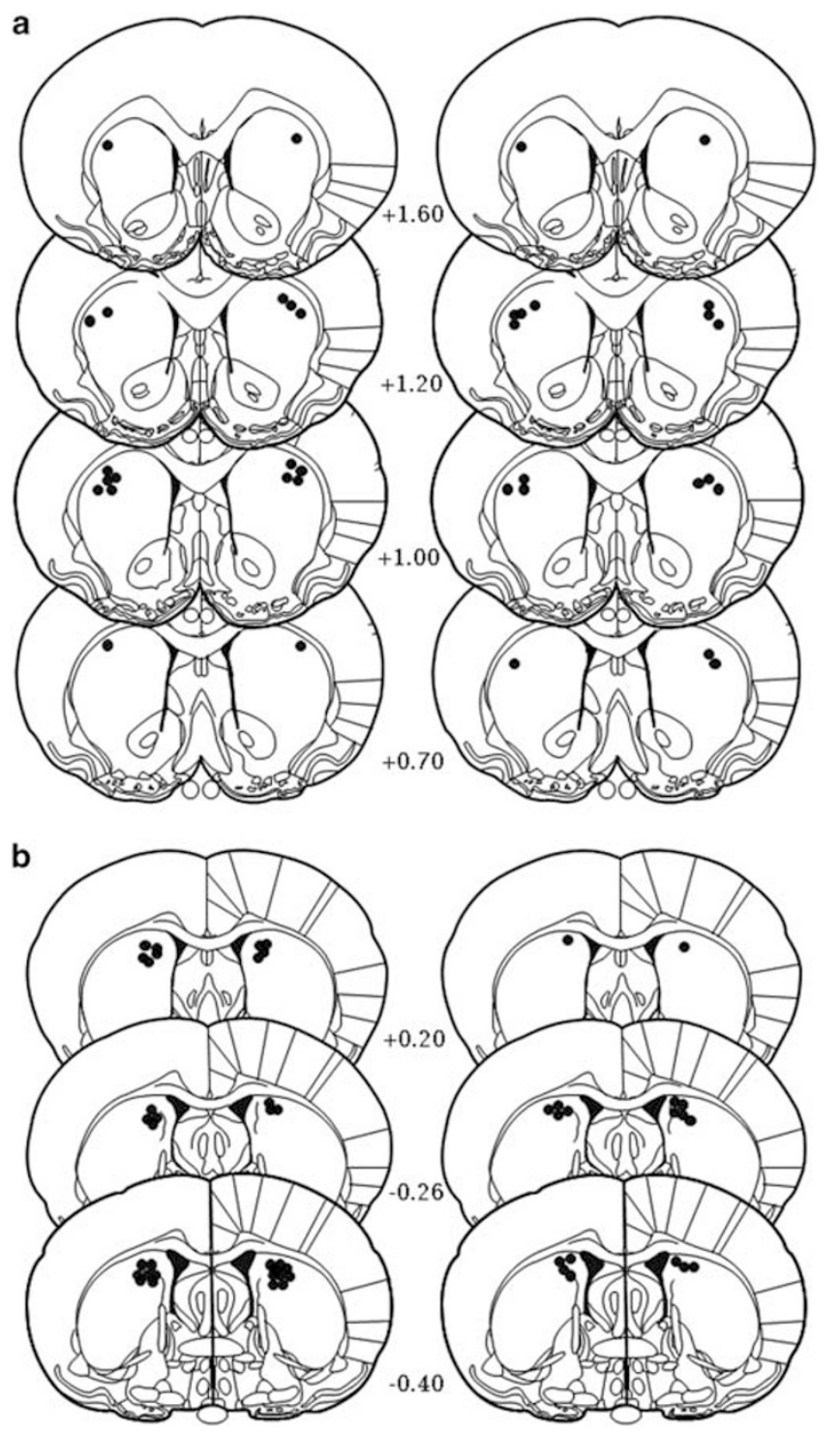

Figure 2 Schematic representations of the localization of injection sites for guide cannulae placed in the aDLS (a) and the pDMS (b) for rats in the second-order group (left) and the FRI control group (right).

\section{Acquisition and Training for Second-Order and FR1 Control Groups}

The mean number and range of cocaine infusions and CS presentations received for each group during every training and testing session throughout the experiments are reported in Supplementary Tables 1-5.

Experiment 1: Bilateral Dopamine Receptor Antagonist Infusions into the pDMS But Not the aDLS Reduced Early Performance of Cocaine Seeking

Cocaine seeking during the 15-min early-performance tests (before cocaine was self-administered in that session) was significantly reduced by dopamine receptor blockade in the pDMS but not in the aDLS. Thus, bilateral infusions of $\alpha$-flupenthixol into the aDLS (Figure 3a; left panel) had no 
a
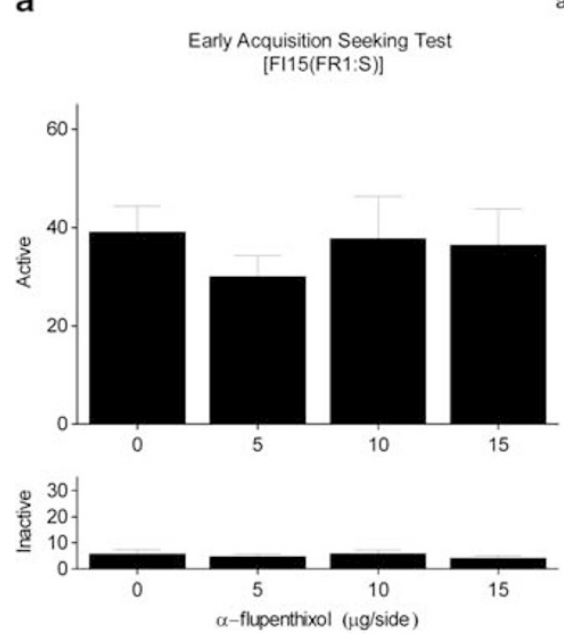

b
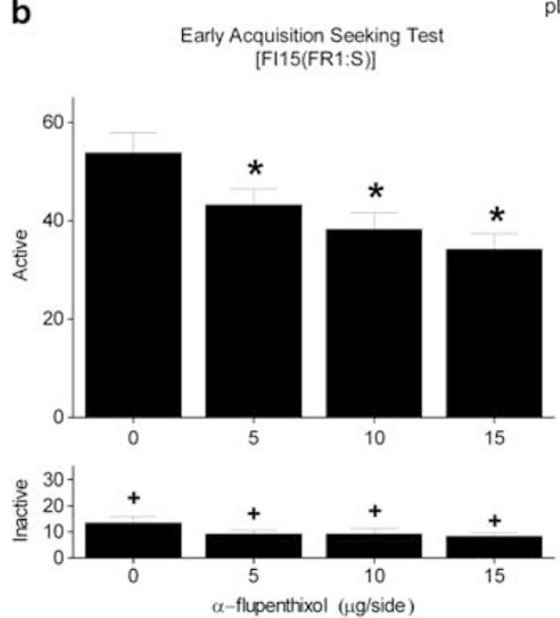

aDLS
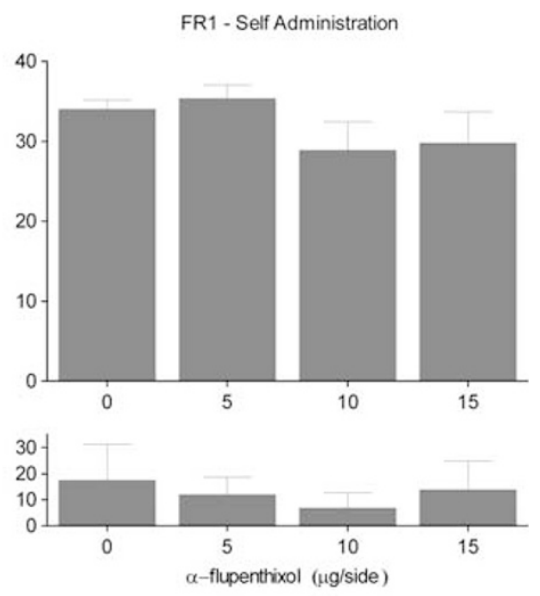

pDMS

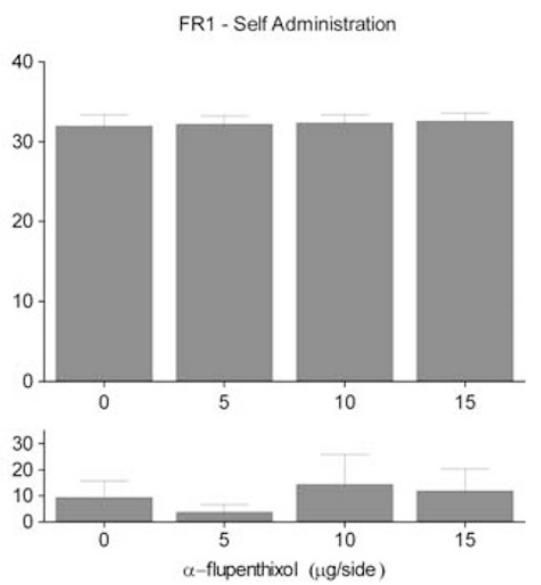

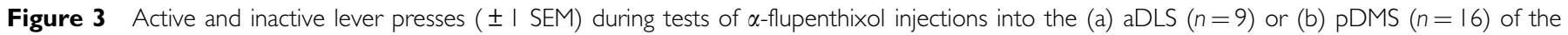

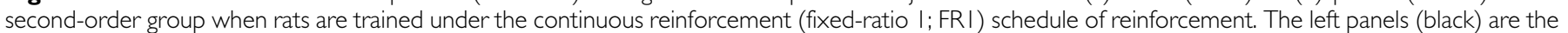

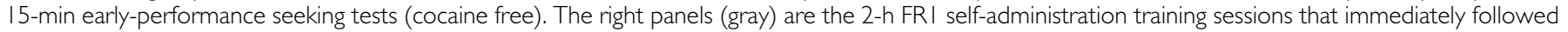

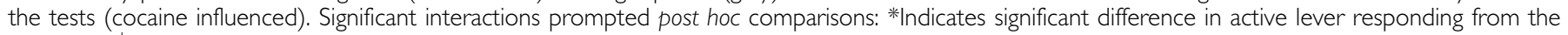
$0-\mu g$ test. ${ }^{+}$Indicates significant within-dose difference between active and inactive lever responses.

effect at this stage, whereas infusions into the pDMS (Figure 3b; left panel) dose dependently decreased active, but not inactive, lever pressing for the cocaine-associated conditioned reinforcer. In the aDLS group, there was a main effect of Lever $(F(1,8)=52.79, p<0.001)$ but no effect of Dose $(F(3,24)=1.00, p=0.409)$ or Lever $\times$ Dose interaction $(F<1)$. In the pDMS group, there were main effects of Lever $(F(1,15)=159.79, \quad p<0.001), \quad$ Dose $\quad(F(3,45)=8.37$, $p<0.001)$, and a Lever $\times$ Dose interaction $(F(3,45)=5.56$, $p=0.002$ ) with all doses of $\alpha$-flupenthixol reducing activelever responding relative to vehicle $(\mathrm{HSD}=8.67)$.

There was no effect of $\alpha$-flupenthixol infusion into either region (Figure $3 a$ and $b$; right panels) on cocaine selfadministration in the session (under FR1) immediately following the 15-min cocaine-seeking test. Active lever pressing remained higher than inactive lever pressing regardless of the test dose as shown by main effects of Lever (aDLS: $\quad F(1,8)=150.89, \quad p<0.001$; $\quad$ pDMS: $F(1,15)=11.98, p=0.003)$ and lack of main effect of Dose or Lever $\times$ Dose interactions (aDLS: $F s(3,24 \leqslant 1.85$, $p s \geqslant 0.165 ;$ pDMS: $F s(3,45) \leqslant 1.02, p s \geqslant 0.391)$.
Experiment 2: Bilateral Dopamine Receptor Antagonist Infusions into the aDLS But Not into the pDMS Reduced Well-Established Performance of Cocaine Seeking

Well-established cocaine seeking during the first 15-min interval-before cocaine had been administered in that session - was significantly reduced following $\alpha$-flupenthixol infusion bilaterally into the aDLS but not into the pDMS in rats with extended training under the second-order schedule of cocaine reinforcement (Figure $4 \mathrm{a}$ and b; left panels, respectively). Following infusion into the aDLS, there were main effects of Lever $(F(1,8)=36.07, p<0.001)$, Dose $(F(3,24)=5.04, p=0.008)$, and a Lever $\times$ Dose interaction $(F(3,24)=4.20, p=0.016)$. The 10 and $15 \mu \mathrm{g} / \mathrm{side}$ doses of $\alpha$-flupenthixol reduced cocaine seeking relative to vehicle $(\mathrm{HSD}=81.56)$. In the pDMS group, there was an effect of Lever reflecting higher responding on active than inactive levers $(F(1,15)=55.17, p<0.001)$, but no effect of Dose or Dose $\times$ Lever interaction $(F s<1)$.

During the second interval that followed the first cocaine infusion there was no effect of dopamine receptor blockade 
a

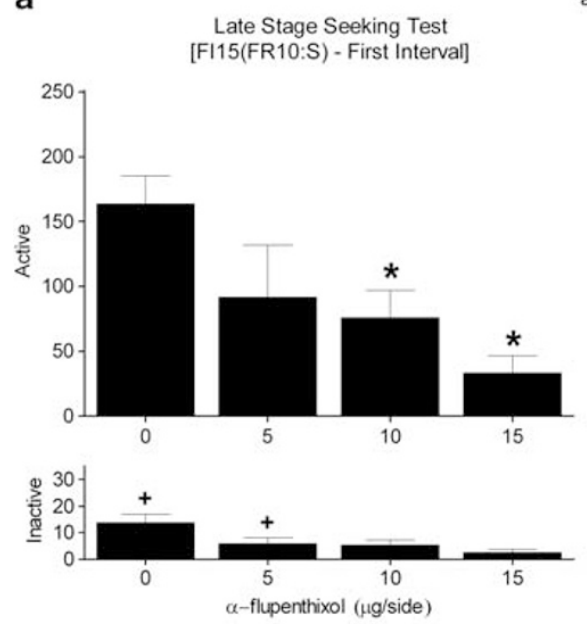

b
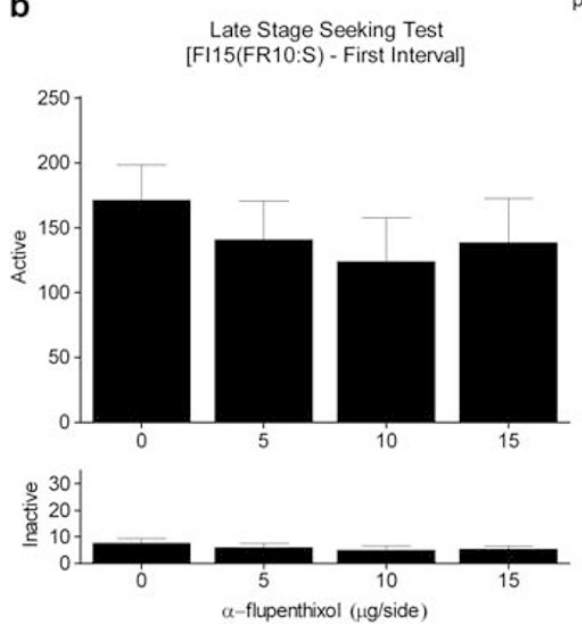

aDLS

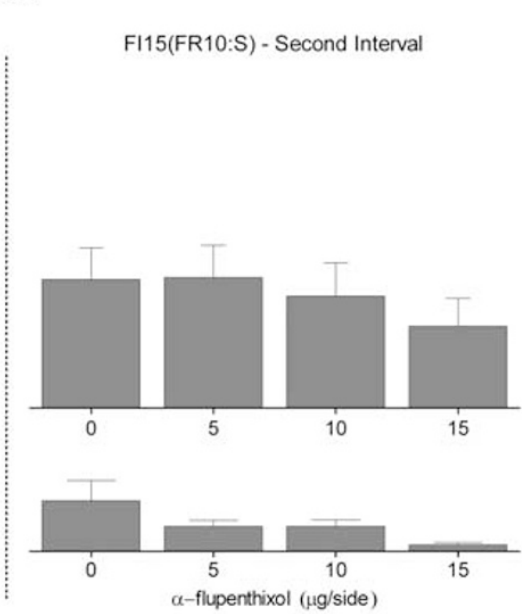

pDMS

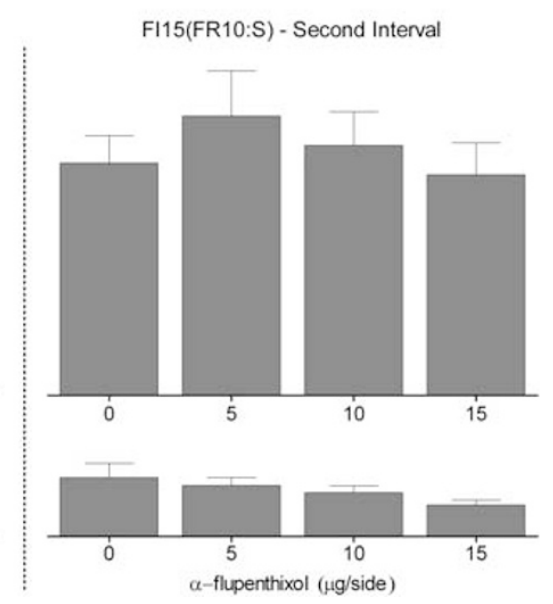

Figure 4 Active and inactive lever presses ( \pm I SEM) during tests of $\alpha$-flupenthixol injections into the (a) aDLS $(n=9)$ or $(b)$ pDMS $(n=16)$ of the second-order group under the second-order [FII5(FRI0:S)] schedule of reinforcement. The left panels (black) are the first interval (cocaine free). The right panels (gray) are the second interval (cocaine influenced). Significant interactions prompted post hoc comparisons: * Indicates significant difference in active lever responding from the 0 - $\mu$ g test. ${ }^{+}$Indicates significant within-dose difference between active and inactive lever responses.

in either brain region (Figure $4 \mathrm{a}$ and $\mathrm{b}$; right panels). Active lever pressing remained higher than inactive lever pressing regardless of the test dose as shown by main effects of Lever (aDLS: $F(1,8)=23.87, p=0.001$; pDMS: $F(1,15)=61.18$, $p<0.001)$ and no effects of Dose or Lever $\times$ Dose interactions $\quad(a D L S: \quad F s(3,24) \leqslant 1.23, \quad p s \geqslant 0.321$; $\quad$ pDMS: Fs $(3,45) \leqslant 1.24, p s \geqslant 0.305)$.

Experiment 3: Bilateral Dopamine Receptor Antagonist Infusions into the pDMS But Not into the aDLS Reduced Performance of Cocaine Seeking Following Both Limited and Extended Cocaine Self-Administration History Under Continuous Reinforcement

In the FR1 control group, cocaine seeking during the 15-min early-performance tests was again unaffected by $\alpha$-flupenthixol infusions into the aDLS, but was significantly reduced following infusions into the pDMS (Figure $5 \mathrm{a}$ and $\mathrm{b}$; left panels, respectively), replicating the results of Experiment 1. For the aDLS group, there was a main effect of Lever $(F(1,8)=52.04, p<0.001)$ but no effect of Dose $(F<1)$ or Lever $\times$ Dose interaction $(F<1)$. For the
pDMS group, there were main effects of Lever $(F(1,8)$ $=74.31, p<0.001)$, Dose $(F(3,24)=8.37, p=0.003)$, and a Lever $\times$ Dose interaction $(F(3,24)=3.33, p=0.038)$, with 10 and $15 \mu \mathrm{g} /$ side $\alpha$-flupenthixol reducing active-lever responding relative to vehicle $(\mathrm{HSD}=16.09)$.

There was no effect of $\alpha$-flupenthixol infusion into either region (Figure $5 \mathrm{a}$ and $\mathrm{b}$; right panels) on cocaine selfadministration in the session (under FR1) following the 15-min early-performance cocaine-seeking tests. Active lever pressing remained higher than inactive lever pressing regardless of the test dose of $\alpha$-flupenthixol as shown by main effects of Lever (aDLS: $F(1,8)=608.42, p<0.001$; pDMS: $F(1,8)=67.90, p<0.001)$. There was a main effect of Dose in the aDLS group $(F(3,24)=3.55, p=0.029)$, but there were no significant post hoc differences $(\mathrm{HSD}=6.23)$. There was no effect of Dose in the pDMS group $(F<1)$ and lack of Lever $\times$ Dose interactions in both groups (aDLS: $F(3,24)=2.24, p=0.110$; pDMS: $F<1)$.

Following the extended self-administration under the FR1 schedule that equated cocaine intake with that of rats in Experiment 1 but with no history of conditioned reinforcement, the effects of aDLS and pDMS $\alpha$-flupenthixol infusions 
a
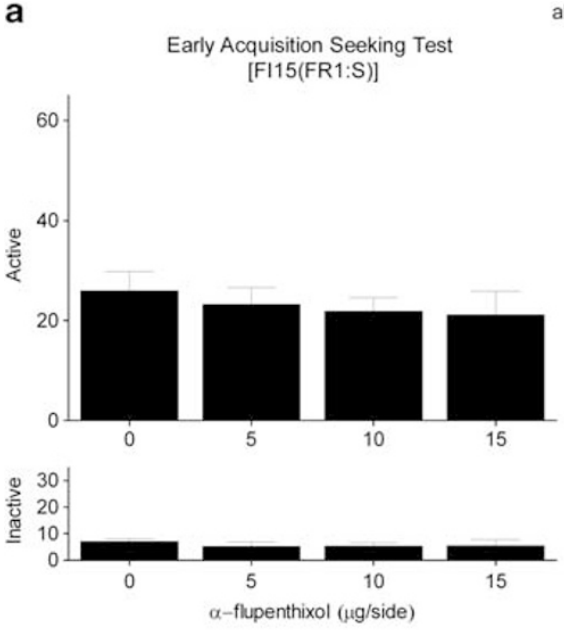

b Early Acquisition Seeking Test
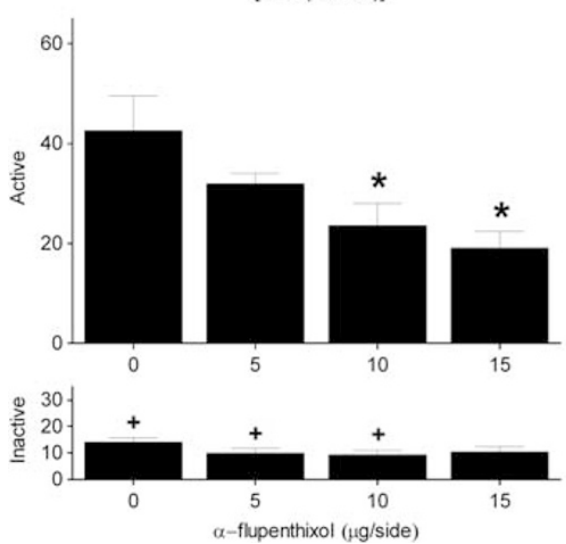

aDLS
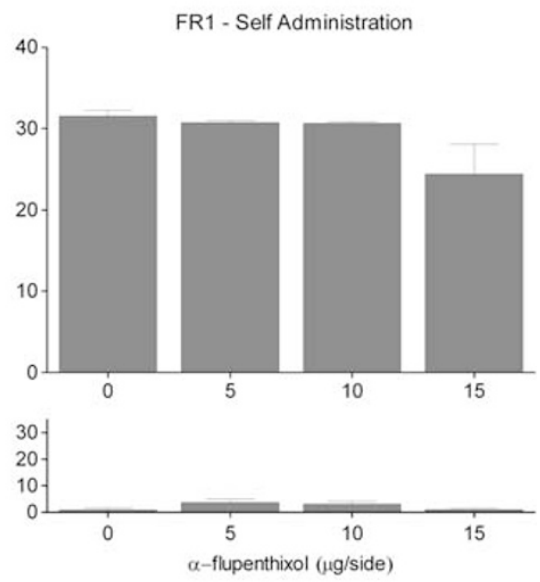

pDMS

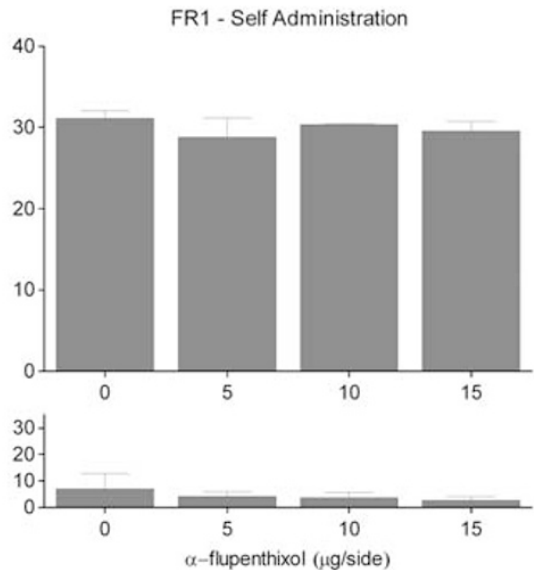

Figure 5 Active and inactive lever presses ( \pm I SEM) during tests of $\alpha$-flupenthixol injections into the $(a)$ aDLS ( $n=9$ ) or (b) pDMS ( $n=9$ ) of the FR I control group when rats are trained under the FRI schedule of reinforcement. The left panels (black) are the I5-min early-performance seeking tests (cocaine free). The right panels (gray) are the FRI self-administration training sessions that immediately followed the tests (cocaine influenced). Significant interactions prompted post hoc comparisons: * ${ }^{*}$ ndicates significant difference in active lever responding from the 0 - $\mu$ g test. ${ }^{+}$Indicates significant within-dose difference between active and inactive lever responses.

were identical to those seen at the early-performance assessment of cue-controlled cocaine seeking. Thus, there was no effect of dopamine receptor blockade in the aDLS, but a significant reduction in active-lever responding when $\alpha$-flupenthixol was infused into the pDMS (Figure 6a and b; left panels, respectively). In the aDLS group, there was a main effect of Lever $(F(1,8)=25.18, p=0.001)$ but no effects of Dose or Lever $\times$ Dose interaction $(F s<1)$. In the pDMS group, there was a main effect of Lever $(F(1,8)=24.28$, $p=0.001)$, Dose $(F(3,24)=4.08, p=0.018)$, and a Lever $\times$ Dose interaction $(F(3,24)=3.21, p=0.041)$, with the $15 \mu \mathrm{g} /$ side $\alpha$-flupenthixol reduced responding relative to vehicle ( $\mathrm{HSD}=19.78)$.

There was no effect of $\alpha$-flupenthixol infusions into either aDLS or pDMS (Figure 6a and b; right panels) on cocaine self-administration in the session (under FR1) following the 15-min cocaine-seeking test. Active-lever pressing remained higher than inactive-lever pressing regardless of the test dose as shown by main effects of Lever (aDLS: $F(1,8)=135.14, p<0.001$; pDMS: $F(1,8)=90.16, p<0.001)$ and no effects of Dose or Lever $\times$ Dose interactions $(F s<1)$.

\section{DISCUSSION}

These results have revealed a double dissociation in the functional involvement of posterior dorsomedial and anterior dorsolateral striatal dopamine transmission in early- or late-stage performance of cocaine-seeking behavior. Bilateral infusions of the dopamine receptor antagonist $\alpha$-flupenthixol into the pDMS dose dependently impaired cocaine seeking only during early-stage tests, having no effect when infused after extended training. In contrast, $\alpha$-flupenthixol infusions into the aDLS had no effect during early, but greatly reduced well-established, cuecontrolled cocaine seeking, confirming our earlier findings of dose-dependent reductions in responding after extended training on the second-order schedule (Belin and Everitt, 2008; Vanderschuren et al, 2005). These effects on acquisition and performance could not be attributed to $\alpha$-flupenthixol-induced changes in cocaine reinforcement (Veeneman et al, 2012), both because the effects on seeking were measured before any influence of self-administered cocaine infusion in each test session and also because 
a
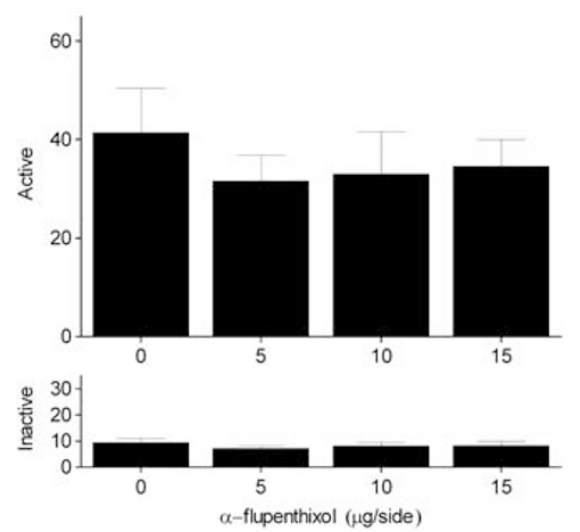

b
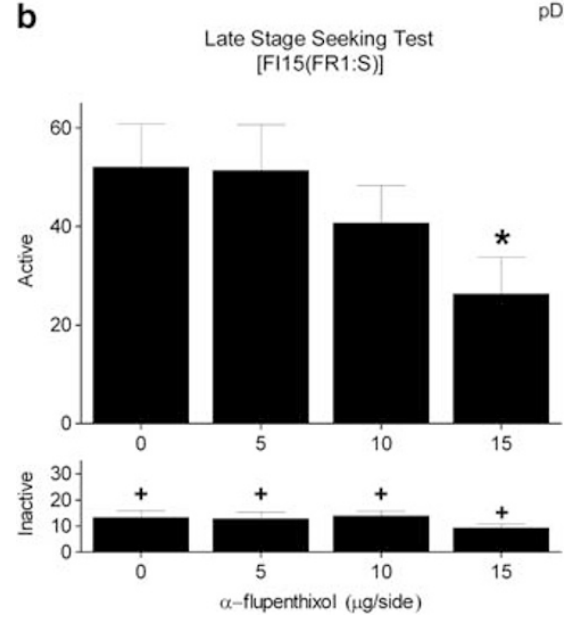

aDLS

FR1 Late - Self Administration
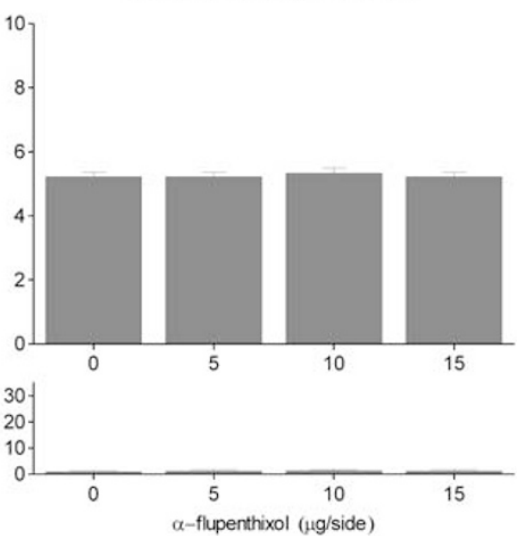

pDMS

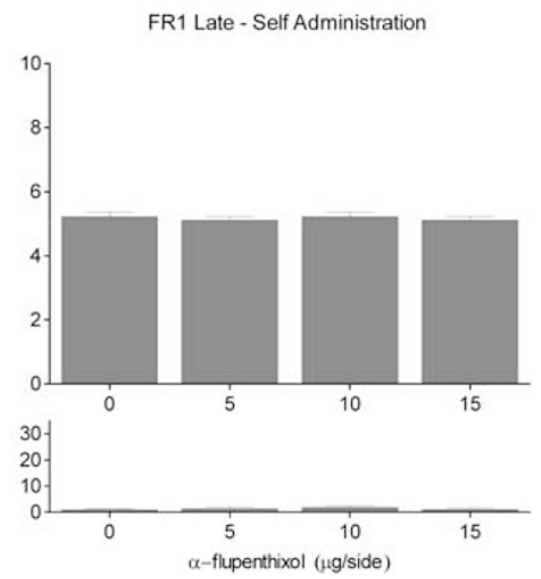

Figure 6 Active and inactive lever presses ( \pm I SEM) during tests with $\alpha$-flupenthixol injections into the $(a)$ aDLS $(n=9)$ or $(b)$ pDMS $(n=9)$ of the FR I control group trained under the FRI schedule of reinforcement with a limit of five cocaine infusions. The left panels (black) are the I5-min seeking tests (cocaine free). The right panels (gray) are the FRI self-administration training sessions that immediately followed the tests (cocaine influenced). Significant

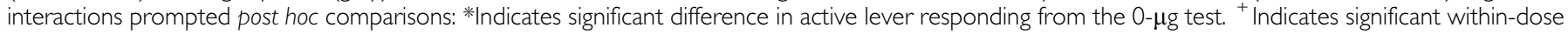
difference between active and inactive lever responses.

$\alpha$-flupenthixol had no measurable effect on taking (early stage) or seeking (late stage) responses after cocaine had been self-administered. The apparent lack of effect of intradorsal striatal infusion of $\alpha$-flupenthixol, which may be seen as inconsistent with that of Veeneman et al (2012), reflects the imposition of a maximum number of infusions per session and thus a ceiling limit on the effect of the dopamine receptor antagonist. Furthermore, the effects of striatal dopamine receptor blockade cannot be accounted for by differential response rates at different test stages during the experiment (Supplementary Experiment). Finally, the shift from pDMS to aDLS control over responding did not simply reflect the impact of a long history of cocaine self-administration, since matching the total cocaine intake to that of the extended training group, but under an FR1 schedule, before the seeking tests resulted in the same sensitivity to pDMS infusions of $\alpha$-flupenthixol as was seen after a short cocaine-taking history. Thus, the shift from pDMS to aDLS control over cocaine-seeking results from an interaction between cocaine taking and extended training under an instrumental seeking schedule that facilitates the emergence of habitual control over behavior (Belin et al, 2009; Dickinson, 1985).

The progressive, dopamine-dependent recruitment of DLS control over cocaine seeking observed here is consistent with previous demonstrations that well-established cue-controlled cocaine seeking is both correlated with increased extracellular dopamine levels in the DLS (Ito et al, 2002) and greatly impaired by dopamine receptor blockade in the aDLS (Belin and Everitt, 2008; Vanderschuren et al, 2005). This has been hypothesized to reflect the gradual consolidation of cocaine seeking as an S-R habit (Everitt and Robbins, 2005), a hypothesis supported by the demonstration that after protracted, but not limited, training, cocaine seeking is resistant to reinforcer devaluation that can be reinstated by transient inactivation of the aDLS (Zapata et al, 2010), thereby re-establishing goal-directedness of this behavior as seen earlier in training (Olmstead et al, 2001). Furthermore, rats trained extensively to self-administer alcohol also show clear evidence of habitual responding in that, after extended training, their behavior was resistant to reinforcer 
devaluation and under DLS control because DLS inactivation rendered rats sensitive again to devaluation. In contrast, at an earlier stage of training, DMS inactivation resulted in habitual responding insensitive to reinforcer devaluation (Corbit et al, 2012).

The present study also extends our understanding of the psychobiological processes that are engaged during an extended cocaine-seeking history and establishes some parallels with the seeking of natural rewards. Thus, the early acquisition and performance of goal-directed responding for an ingestive reinforcer depends upon the pDMS whereas, habitual behavior, which develops after overtraining, depends upon the aDLS (Balleine et al, 2009). Furthermore, in instrumental conditioning, both NMDA receptor blockade in the pDMS (Yin et al, 2005) and disruption of ERK signalling in the pDMS, but not in the aDLS, prevent A-O learning during the acquisition of foodreinforced responding, resulting in insensitivity to outcome devaluation (Shiflett et al, 2010). Conversely, in overtrained rats, lesions, inactivation (Yin et al, 2004) or dopaminergic denervation (Faure et al, 2005) of the aDLS disrupts both performance and establishment of S-R control over behavior. This pDMS to aDLS shift in the locus of control over behavior has also been shown in spatial navigation tasks to reflect the transition from flexible, adaptive to inflexible, habitual performance (Packard and McGaugh, 1996; Lex et al, 2011). While responding for sucrose or other ingestive reinforcers also shifts from goal-directed to stimulus-response, or habitual, control (Adams and Dickinson, 1981), it has also been demonstrated that this transition occurs more slowly than is the case when rats are responding for alcohol (Dickinson et al, 2002). Moreover, responding for sucrose became insensitive to devaluation sooner in rats given non-contingent exposure to alcohol (Corbit et al, 2012), perhaps suggesting an augmentation of the dopaminergic circuitry connecting striatal subregions.

The pDMS to DLS shift in the control over cocaine seeking can be integrated within a broader striatal dynamics model, based on a shift from the ventral to the dorsal striatum that has been suggested to account for the development of cocaine-seeking habits (Belin and Everitt, 2008; Belin et al, 2009; Everitt and Robbins, 2005). Thus, in addition to the role of dopamine transmission in the Acb and associated ventral striatal areas in mediating the reinforcing effects of addictive drugs (Suto et al, 2009, 2010; Ikemoto, 2007), hence supporting the acquisition of drug self-administration (eg, Roberts and Koob, 1982), the AcbC (Ito et al, 2004) and its functional interactions with the basolateral amygdala (Whitelaw et al, 1996; Di Ciano and Everitt, 2004) additionally mediate the impact of drugassociated conditioned reinforcers on the acquisition of cocaine-seeking behavior (Everitt and Robbins, 2000; Schindler et al, 1988) and the tolerance of delays to primary reinforcement (Cardinal et al, 2002).

This AcbC involvement in the acquisition and early performance of cocaine seeking is consistent with the demonstration that glutamate-dopamine co-activation (Smith-Roe and Kelley, 2000) and protein synthesis (Hernandez et al, 2002) within the AcbC are apparently required during the acquisition of instrumental responding. Additionally, evidence from computational modelling and functional imaging studies have provided further evidence that ventral striatal processes may drive nigro-dorsal striatal activity to guide decision making (Kahnt et al, 2009). These data, taken together with the present results, suggest that a distributed ventral striatal-posterior dorsomedial striatal network is engaged in the acquisition and early performance of cue-controlled cocaine seeking under A-O control. They conform to the model that the ventral striatum, with its dopaminergic inputs from the VTA, acts as a 'critic', learning to predict future rewards, whereas the dorsal striatal 'actor' maintains information about the outcomes to optimize decision making and instrumental performance (O’Doherty et al, 2004).

In addition to demonstrating a role for the pDMS in the acquisition of cocaine seeking, the present study provides further evidence that this behavior becomes more dependent on aDLS dopaminergic mechanisms over time (Belin and Everitt, 2008; Vanderschuren et al, 2005; Zapata et al, 2010). The progressive recruitment of the aDLS by prolonged selfadministration of psychostimulants has also been demonstrated in terms of cellular plasticity following chronic stimulant drug administration (Jedynak et al, 2007), as well as metabolic and dopaminergic neuroadaptations (Porrino et al, 2007). Thus, in monkeys self-administering cocaine over an extended, but not a brief, time period, adaptations in the dopamine transporter and D2 dopamine receptors, and metabolic markers that are initially restricted to more ventral and posterior parts of the striatum, become increasingly prominent in the dorsal and lateral anterior striatum (Letchworth et al, 2001; Porrino et al, 2004). Moreover, increased extracellular dopamine in the DLS, but not in the AcbS or AcbC, is seen following several weeks of drug seeking experience under the second-order schedule used here (Ito et al, 2000). These experimental data resonate well with the demonstration that cue-induced craving in human cocaine addicts is associated with general activation of, and increased dopamine transmission within, the dorsal striatum (Garavan et al, 2000; Volkow et al, 2006; Wong et al, 2006).

The ventral-to-dorsal transition in the striatal locus of control over cocaine seeking has been hypothesized (Everitt and Robbins, 2005; Belin and Everitt, 2008) to be mediated by striato-nigro-striatal ascending, or 'spiralling' dopaminedependent circuitry that functionally links domains of the ventral and dorsal striatum (Haber, 2003; Ikemoto, 2007). We have previously demonstrated the functional importance of this link by disconnecting the AcbC and the DLS by infusing $\alpha$-flupenthixol into the aDLS contralateral to a lesion of the AcbC. This disconnection resulted in an identical degree of impairment of well-established cocaine seeking to that observed after bilateral infusions of $\alpha$ flupenthixol into the aDLS (present results; Belin and Everitt, 2008; Vanderschuren et al, 2005). Thus, the longterm performance of instrumental seeking responses for cocaine depends upon a distributed striatal network involving interactions between the AcbC and the aDLS, but not the pDMS. Taken together with the current results, it can be suggested that a shift in the striatal network control over instrumental seeking behavior occurs from AcbC-pDMS early, to the AcbC-aDLS when well established and habitual.

This view of dynamic transitions within the striatum is in agreement with the demonstration that the development of 
habitual control over behavior is associated with online transitions in electrophysiological activity between the DMS and DLS, such that DMS neuronal activity is actively engaged during acquisition and early behavioral performance, then progressively decreases over training, eventually leaving DLS activity to drive both habitual performance in a T-maze task in rats (Thorn et al, 2010) or enhance motor performance on a rotarod in mice (Yin et al, 2009). Furthermore, based on the evidence that the functional activity in the pDMS declines, eventually to disappear, when habitual performance is driven by the DLS (Kahnt et al, 2009) and also the demonstration that the DLS, unlike the pDMS, does not receive direct inputs from the BLA (Kelley et al, 1982), we suggest that wellestablished cocaine seeking, which is highly dependent upon contingent presentation of cocaine-associated CSs acting as conditioned reinforcers (Everitt and Robbins, 2000), is subserved by a BLA-AcbC-aDLS network, coordinated by recurrent, ascending striato-nigro-striatal circuitry (Gerfen et al, 1987; Haber, 2003; Ikemoto, 2007; Nauta et al, 1978). Thus, in the course of cocaine addiction, a progressive transition may occur in the neural and psychological mechanisms governing drug seeking, with the AcbC-pDMS network controlling goal-directed cocaine seeking eventually becoming subordinate to AcbC-aDLS circuitry when drug seeking is established as a maladaptive, incentive habit (see Belin et al, 2009).

\section{ACKNOWLEDGEMENTS}

We thank Dr Yann Pelloux and Dr Gonzalo Urcelay for their valuable suggestions as well as Alan Lyon and David Theobald for their technical assistance. This work was supported by United Kingdom Medical Research Council (MRC) Grants 9536855 and 1002231 to BJE and was conducted within the MRC/Wellcome Trust Behavioral and Clinical Neuroscience Institute. DB was supported by the INSERM and the IREB.

\section{DISCLOSURE}

The authors declare no conflicts of interest. Over the past three years DB has received compensation from AB Science for services that are unrelated to the present study.

\section{REFERENCES}

Adams CD, Dickinson A (1981). Instrumental responding following reinforcer devaluation. Q J Exp Psychol B 33: 109-122.

Balleine BW, Liljeholm M, Ostlund SB (2009). The integrative function of the basal ganglia in instrumental conditioning. Behav Brain Res 199: 43-52.

Belin D, Economidou D, Pelloux Y, Everitt BJ (2011). Habit formation and compulsion. Neuromethods 53: 337-378.

Belin D, Everitt BJ (2008). Cocaine seeking habits depend upon dopamine-dependent serial connectivity linking the ventral with the dorsal striatum. Neuron 57: 432-441.

Belin D, Jonkman S, Dickinson A, Robbins TW, Everitt BJ (2009). Parallel and interactive learning processes within the basal ganglia: relevance for the understanding of addiction. Behav Brain Res 199: 89-102.

Cardinal RN, Parkinson JA, Lachenal G, Halkerston KM, Rudarakanchana N, Hall J et al (2002). Effects of selective excitotoxic lesions of the nucleus accumbens core, anterior cingulate cortex, and central nucleus of the amygdala on autoshaping performance in rats. Behav Neurosci 116: 553-567.

Cardinal RN, Pennicott DR, Sugathapala CL, Robbins TW, Everitt BJ (2001). Impulsive choice induced in rats by lesions of the nucleus accumbens core. Science 292: 2499-2501.

Corbit LH, Nie H, Janak PH (2012). Habitual alcohol seeking: time course and the contribution of subregions of the dorsal striatum. Biol Psychiatry. http://dx.doi.org/10.1016/j.biopsych. 2012.02.02.

Di Ciano P, Everitt BJ (2004). Direct interactions between the basolateral amygdala and nucleus accumbens core underlie cocaine-seeking behaviour by rats. J Neurosci 24: 7167-7173.

Dickinson A (1985). Actions and habits: the development of behavioural autonomy. Phil Trans $R$ Soc Lond B 308: 67-78.

Dickinson A, Wood N, Smith JW (2002). Alcohol seeking by rats: action or habit? Q J Exp Psychol B 55: 331-348.

Everitt BJ, Belin D, Economidou D, Pelloux Y, Dalley JW, Robbins TW (2008). Neural mechanisms underlying the vulnerability to develop compulsive drug-seeking habits and addiction. Phil Trans $R$ Soc B 363: 3125-3135.

Everitt BJ, Robbins TW (2000). Second-order schedules of drug reinforcement in rats and monkeys: measurement of reinforcing efficacy and drug-seeking behaviour. Psychopharmacology 153: 17-30.

Everitt BJ, Robbins TW (2005). Neural systems of reinforcement for drug addiction: from actions to habits to compulsion. Nat Neurosci 8: 1481-1489.

Faure A, Haberland U, Condé F, El Massioui N (2005). Lesion to the nigrostriatal dopamine system disrupts stimulus-response habit formation. J Neurosci 25: 2771-2780.

Garavan H, Pankiewicz J, Bloom A, Cho JK, Sperry L, Ross TJ et al (2000). Cue-induced cocaine craving: neuroanatomical specificity for drug users and drug stimuli. Am J Psychiatry 157: 1789-1798.

Gerfen CR, Herkenham M, Thibault J (1987). The neostriatal mosaic: II. patch- and matrix-directed mesostriatal dopaminergic and non-dopaminergic systems. J Neurosci 7: 3915-3934.

Haber SN (2003). The primate basal ganglia: parallel and integrative networks. J Chem Neuroanat 26: 317-330.

Hernandez PJ, Sadeghian K, Kelley AE (2002). Early consolidation of instrumental learning requires protein synthesis in the nucleus accumbens. Nat Neurosci 5: 1327-1331.

Ikemoto S (2007). Dopamine reward circuitry: two projection systems from the ventral midbrain to the nucleus accumbensolfactory tubercle complex. Brain Res Rev 56: 27-78.

Ito R, Dalley JW, Howes SR, Robbins TW, Everitt BJ (2000). Dissociation in conditioned dopamine release in the nucleus accumbens core and shell in response to cocaine cues and during cocaine-seeking behaviour in rats. J Neurosci 20: 7489-7495.

Ito R, Dalley JW, Robbins TW, Everitt BJ (2002). Dopamine release in the dorsal striatum during cocaine-seeking behaviour under the control of a drug-associated cue. J Neurosci 22: 6247-6253.

Ito R, Robbins TW, Everitt BJ (2004). Differential control over cocaine-seeking behaviour by nucleus accumbens core and shell. Nat Neurosci 7: 389-397.

Jedynak JR, Uslaner JM, Esteban JA, Robinson TE (2007). Methamphetamine-induced structural plasticity in the dorsal striatum. Eur J Neurosci 25: 847-853.

Kahnt T, Park SQ, Cohen MX, Beck A, Heinz A, Wrase J (2009). Dorsal striatal-midbrain connectivity in humans predicts how reinforcements are used to guide decisions. J Cogn Neurosci 21: 1332-1345.

Kelley AE, Domesick VB, Nauta WJ (1982). The amygdalostriatal projection in the rat - an anatomical study by anterograde and retrograde tracing methods. Neuroscience 7: 615-630.

Letchworth SR, Nader MA, Smith HR, Friedman DP, Porrino LJ (2001). Progression of changes in dopamine transporter binding 
site density as a result of cocaine self-administration in rhesus monkeys. J Neurosci 21: 2799-2807.

Lex B, Hauber W (2010). The role of dopamine in the prelimbic cortex and the dorsomedial striatum in instrumental conditioning. Cereb Cortex 20: 873-883.

Lex B, Sommer S, Hauber W (2011). The role of dopamine in the dorsomedial striatum in place and response learning. Neuroscience 172: 212-218.

Murray JE, Everitt BJ, Belin D (2012). N-Acetylcysteine reduces early- and late-stage cocaine seeking without affecting cocaine taking in rats. Addict Biol 17: 437-440.

Nauta WJH, Smith GP, Faull RLM, Domesick VB (1978). Efferent connections and nigral afferents of the nucleus accumbens septi in the rat. Neuroscience 3: 385-401.

O’Doherty J, Dayan P, Schultz J, Deichmann R, Friston K, Dolan RJ (2004). Dissociable roles of ventral and dorsal striatum in instrumental conditioning. Science 304: 452-454.

Olmstead MC, Lafond MV, Everitt BJ, Dickinson A (2001). Cocaine seeking by rats is a goal-directed action. Behav Neurosci 115: 394-402.

Packard MG, McGaugh JL (1996). Inactivation of hippocampus or caudate nucleus with lidocaine differentially affects expression of place and response learning. Neurobiol Learn Mem 65: 65-72.

Paxinos G, Watson C (1998). The Rat Brain in Stereotaxic Coordinates, 4th Edn. Academic Press: San Diego, CA.

Porrino LJ, Lyons D, Smith HR, Daunais JB, Nader MA (2004). Cocaine self-administration produces a progressive involvement of limbic, association, and sensoriomotor striatal domains. J Neurosci 24: 3554-3562.

Porrino LJ, Smith HR, Nader MA, Beveridge TJ (2007). The effects of cocaine: a shifting target over the course of addiction. Prog Neuropsychopharmacol Biol Psychiatry 31: 1593-1600.

Roberts DC, Koob GF (1982). Disruption of cocaine self-administration following 6-hydroxydopamine lesions of the ventral tegmental area in rats. Pharmacol Biochem Behav 17: 901-904.

Robledo P, Maldonado-Lopez R, Koob GF (1992). Role of dopamine receptors in the nucleus accumbens in the rewarding properties of cocaine. Ann NY Acad Sci 654: 509-512.

Schindler CW, Katz JL, Goldberg SR (1988). The use of secondorder schedules to study the influence of environmental stimuli on drug-seeking behaviour. NIDA Res Monogr 84: 180-195.

Shiflett MW, Brown RA, Balleine BW (2010). Acquisition and performance of goal-directed instrumental actions depends on ERK signalling in distinct regions of dorsal striatum of rats. J Neurosci 30: 2951-2959.

Smith-Roe SL, Kelley AE (2000). Coincident activation of NMDA and dopamine D1 receptors within the nucleus accumbens core is required for appetitive instrumental learning. J Neurosci 20: 7737-7742.

Suto N, Ecke LE, Wise RA (2009). Control of within-binge cocaineseeking by dopamine and glutamate in the core of nucleus accumbens. Psychopharmacology 205: 431-439.
Suto N, Ecke LE, You ZB, Wise RA (2010). Extracellular fluctuations of dopamine and glutamate in the nucleus accumbens core and shell associated with lever-pressing during cocaine self-administration, extinction, and yoked cocaine administration. Psychopharmacology 211: 267-275.

Thorn CA, Atallah H, Howe M, Graybiel AM (2010). Differential dynamics of activity changes in dorsolateral and dorsomedial striatal loops during learning. Neuron 66: 781-795.

Vanderschuren LJMJ, Di Ciano P, Everitt BJ (2005). Involvement of the dorsal striatum in cue-controlled cocaine seeking. J Neurosci 25: 8665-8670.

Veeneman MM, Broekhoven MH, Damsteegt R, Vanderschuren LJ (2012). Distinct contributions of dopamine in the dorsolateral striatum and nucleus accumbens shell to the reinforcing properties of cocaine. Neuropsychopharmacology 37: 487-498.

Volkow ND, Wang G-J, Telang F, Fowler JS, Logan J, Childress A-R et al (2006). Cocaine cues and dopamine in dorsal striatum: mechanism of craving in cocaine addiction. J Neurosci 26: 6583-6588.

Whitelaw RB, Markou A, Robbins TW, Everitt BJ (1996). Excitotoxic lesions of the basolateral amygdala impair the acquisition of cocaine-seeking behaviour under a second-order schedule of reinforcement. Psychopharmacology 127: 213-224.

Wise RA (2004). Dopamine, learning and motivation. Nat Rev Neurosci 5: 483-494.

Wong DF, Kuwabara H, Schretlen DJ, Bonson KR, Zhou Y, Nandi A et al (2006). Increased occupancy of dopamine receptors in human striatum during cue-elicited cocaine craving. Neuropsychopharmacology 31: 2716-2727.

Wyvell CL, Berridge KC (2000). Intra-accumbens amphetamine increases the conditioned incentive salience of sucrose reward: enhancement of rewarding "wanting" without enhancing "liking" or response reinforcement. J Neurosci 20: 8122-8130.

Yin HH, Knowlton BJ, Balleine BW (2004). Lesions of dorsolateral striatum preserve outcome expectancy but disrupt habit formation in instrumental learning. Eur J Neurosci 19: 181-189.

Yin HH, Mulcare SP, Hilário MRF, Clouse E, Holloway T, Davis MI et al (2009). Dynamic reorganization of striatal circuits during the acquisition and consolidation of a skill. Nat Neurosci 12: 333-341.

Yin HH, Ostlund SB, Knowlton BJ, Balleine BW (2005). The role of the dorsomedial striatum in instrumental conditioning. Eur $J$ Neurosci 22: 513-523.

Zapata A, Minney VL, Shippenberg TS (2010). Shift from goaldirected to habitual cocaine seeking after prolonged experience in rats. J Neurosci 30: 15457-15463.

This work is licensed under the Creative Commons Attribution-NonCommercial-No Derivative Works 3.0 Unported License. To view a copy of this license, visit http://creativecommons.org/ licenses/by-nc-nd/3.0/

Supplementary Information accompanies the paper on the Neuropsychopharmacology website (http://www.nature.com/npp) 